\title{
Notas corológicas sobre la flora vascular del País Vasco y aledaños (XIII).
}

\section{Chorological notes on the vascular flora of the Basque Country and neighbouring territories (XIII).}

\author{
Eneko J. Díaz¹, Javier Elorza1', Sergio González-Ahedo', Eduardo Miguel1, Oreina Orrantia1, \\ Santiago Patino ${ }^{1}$, Amador Prieto ${ }^{1}$, Javier Valencia1 ${ }^{1}$ Begoña Zorrakin ${ }^{1}$
}

*

\section{Resumen}

Se comentan alrededor de 75 taxones de plantas vasculares raras o escasas en la Comunidad Autónoma Vasca (CAPV) y en territorios limítrofes de las cuales la mayoría de ellas han sido localizadas en el territorio histórico de Bizkaia. Un número importante se consideran taxones amenazados, una docena son novedades provinciales de interés y otros muchos cuentan con citas en escasas localidades de la provincia. Entre ellos destacan Antirrhinum braun-blanquetii, Salix cantabrica, Pteris vittata, Epipactis palustris, Dittrichia graveolens, Himantoglossum hircinum, Galega officinalis, Orchis champagneuxii, Orchis militaris o Sorbus hybrida.

Palabras clave: Notas corológicas, flora vizcaína, flora amenazada.

\begin{abstract}
We discuss 75 species of rare vascular plants of the Basque Autonomous Community and bordering territories, the great majority of which have been located within the Province of Bizkaia. A large number of these are considered threatened flora, a dozen are new reports of provincial interest and many others have been reported in very few locations within Bizkaia. Of special interest is the presence of: Antirrhinum braun-blanquetii, Salix cantabrica, Pteris vittata, Epipactis palustris, Dittrichia graveolens, Himantoglossum hircinum, Galega officinalis, Orchis champagneuxii, Orchis militaris or Sorbus hybrida.
\end{abstract}

Key words: Chorological notes, flora of Bizkaia (Spain), threatened flora.

\footnotetext{
1 Sociedad de Ciencias Naturales de Sestao.

Calle Los Baños n 55, Apartado 41, 48910, Sestao (Bizkaia)

*Correspondencia: botanikasestao@gmail.com botanikasestao.blogspot.com
} 


\section{Laburpena}

Guztira Euskal Autonomia Erkidegoan (EAEn) eta inguruetan landare baskular arraro edo eskasak diren 75 taxon aipatzen dira, gehienak Bizkaian aurkituak. Kopuru garrantzitsu bat mehatxu egoeran dauden taxonak dira, dozena bat herrialdean intereseko aipu berriak dira eta beste askok bertako herri gutxitan izan dute aipuren bat. Horien artean, nabarmenenak: Antirrhinum braun-blanquetii, Salix cantabrica, Pteris vittata, Epipactis palustris, Dittrichia graveolens, Himantoglossum hircinum, Galega officinalis, Orchis champagneuxii, Orchis militaris edo Sorbus hybrida.

Gako hitzak: Ohar korologikoak, Bizkaiko flora, flora mehatxatua.

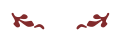

Se continúa con la revisión de materiales recolectados a lo largo de las últimas campañas de herborización, la mayoría de las cuales han sido desarrolladas en los últimos años (2010-16) para la elaboración de diversos proyectos sobre Especies Amenazadas de Bizkaia, y más en concreto para la "Cartografía a escala 1:5000 de las poblaciones vizcaínas de plantas incluidas en el catálogo vasco de especies amenazadas", subvencionada por el Departamento de Medio Ambiente y Política territorial del Gobierno Vasco (Patino et al., 2013). Para la nomenclatura y autoría de los taxones se sigue el criterio de Flora Iberica (Castroviejo et al., 1986-2015).

Se aportan algunos datos de la localidad que se cita: aspectos geográficos, el lugar y hábitat donde se ha encontrado, y en cursiva se indica la persona(s) que aporta la cita y/o datos precisos para su localización por los autores. Para su determinación geográfica se dan las cuadrículas UTM de $1 \mathrm{~km}^{2}$ que se refieren al Datum ETRS89 (EPSG 25830), estando en todos los casos incluidas en el huso 30T.

A continuación se describe el hábitat del taxón, valiéndose principalmente de la obra de Aizpuru et al. (1999), distribución, procedencia y abundancia en la zona, y se hace una breve revisión de citas previas realizadas por los mismos o por otros autores para la mayoría de los taxones que aparecen en este trabajo. Si se tiene conocimiento de su recolección previa se indica entre paréntesis el código del herbario, así como la importancia de la cita. Finalmente se señala, allí donde procede, el estatus de protección de la especie para el País Vasco según Lista Roja de la Flora Vascular de la CAPV (Aizpuru et al., 2010). De aquí en adelante las referencias que se encuentren en el texto a la categoría de protección en Euskadi son las recogidas en dicha publicación.

Los taxones citados aparecen por orden alfabético para facilitar su consulta. Por otro lado, indicar que de buena parte de dichos taxones se conservan pliegos en el Herbario SESTAO (Sociedad de Ciencias Naturales de Sestao). 


\section{TAXONES DE FLORA VASCULAR}

\section{Alopecurus geniculatus L.}

BIZKAIA: 30TVN7491, Karrantza Harana/Nalle de Carranza, Sopeña, 735 m, suelos encharcados, sustrato silíceo, ambiente argomal-helechal, 06.06.2015, E. Miguel, E.J. Díaz, T. Oyanarte, S. Patino \& J. Valencia.

Planta que forma parte de las orlas herbáceas de masas de agua, apareciendo en cunetas encharcadas, orillas de charcas y balsas, en un amplio rango de altitud (0-1100 m).

Desconocida en la actualidad en Bizkaia, Aseginolaza et al. (1984) la citan de la localidad alavesa de Arreo, aportando así mismo dos antiguas referencias para nuestro territorio: Durango (Bubani, 1901) y Elorriaga (Gredilla, 1913). Por lo que la nueva localidad tiene un indudable interés corológico.

\section{Anacyclus radiatus Loisel}

CANTABRIA: 30TVP597133, Noja, Playa de Trengandín, Helgueras, 6 m, terrenos arenosos en la parte posterior de las dunas, junto a la carretera, 31.10.2015, J. Elorza.

Planta de óptimo mediterráneo que aparece puntual en zonas cercanas a la costa en ambientes ruderalizados y posiblemente ligada al transporte de semillas con fines industriales.

Aedo et al. (1993) lo citan de Laredo, donde posteriormente la recolectan J.A. Campos y G. Rodríguez el 05.06.1999. Duran (2014) recoge citas de la franja costera oriental de Cantabria de Aedo et al. (1993), Loriente (1998), MA 693478. Para Bizkaia fue citado de Barakaldo y Valle de Trápaga-Trapagaran (Patino \& Valencia, 2000) y de Zierbena (Patino et al., 2009-2010).

\section{Antirrhinum braun-blanquetii Rothm.}

BIZKAIA: 30TVN6587, Karrantza HaranaNValle de Carranza, Peña El Moro, 768-785 m, rellanos y grietas de lapiaz, 17.07.2013, S. Patino, E.J. Díaz \& F.J. Martínez; 30TVN6586, Karrantza HaranaNalle de Carranza, Peña El Moro, 550 m, prado sobre calizas, 12.05.2015, S. González Ahedo.

Endemismo ibérico, con presencia en graveras y pastos pedregosos, representando la CAPV su límite oriental de distribución. Conocido del vecino Valle de Soba (Cantabria), aparece ahora en territorio vizcaíno de forma discreta, formando pequeñas manchas en rellanos y grietas de lapiaz. Novedad provincial. Este taxón está incluido en la Lista 
Roja de la Flora Vascular de la CAPV bajo el epígrafe "Casi amenazada" (NT). (Fig. S1 en Material Suplementario Online).

Aptenia cordifolia (L. fil.) Schwantes

GIPUZKOA: 30TWN6094, Zumaia, Itzurun, $21 \mathrm{~m}$, cunetas y ambientes ruderales o nitrificados, 15.08.2014, J. Elorza.

Caméfito de la familia Aizoaceae, introducido de Sudáfrica, se cultiva como ornamental y aparece asilvestrado sobre muros, tapias y taludes. Es muy raro en zonas del litoral.

\section{Arctium lappa L.}

NAVARRA: 30TWN6953, Basaburua, Beruete, 575 m, taludes y cunetas de vías de comunicación, 17.08.2015, J. Elorza.

Aparece en herbazales de suelo fresco, cunetas y bordes de camino. En ocasiones se confunde con $A$. minus del que se conocen escasas citas en el tercio septentrional de Navarra (Lorda, 2001 y 2013). A. lappa se localiza muy raramente en nuestro territorio (Fig. S2).

\section{Armeria pubinervis Boiss.}

BIZKAIA: 30TVN6688, Karrantza Harana/Valle de Carranza, Ventana de Relux, 735764 m, 18.6.2014. S. González Ahedo y A. Prieto.

Se trata de un endemismo atlántico montano, que aparece en repisas herbosas de zonas calizas de media montaña atlántica. En Patino et al. (2009-2010) ya se citaba en este entorno, aunque dentro del municipio de Ramales de la Victoria (Cantabria). Se han localizado algunos ejemplares entre las rocas en el karst, ubicadas ya en territorio vizcaíno. Nueva localidad vizcaína para esta especie que cuenta con un par de citas en localidades de la provincia. Este taxón se corresponde con lo que en Aizpuru et al. (1999) se determina como Armeria pubinervis subsp. orissonensis Donad. y está incluido en la Lista Roja de la Flora Vascular CAPV bajo el epígrafe "Casi amenazada" (NT).

\section{Azolla filiculoides Lam}

ÁLAVA: 30TVN9832, Fontecha, río Ebro, 470 m, zonas remansadas del río 20.08.2014, S. Gaspar \& A. Prieto. 
Hasta ahora conocido de al menos 5 cuadrículas UTM de 1 km de lado (Agut, 2012) se han observado en diversos puntos del río Ebro.

Este pteridofito, introducido de América tropical y templada, se naturaliza en aguas remansadas o estancadas. La primera cita para la CAPV se debe a Pedro Uribe-Echebarría que la recolectó en 1998 en Vitoria/Gasteiz, Amarita, presa del río Sta. Engracia. Posteriormente se había localizado hasta en otras cinco cuadrículas UTM de 1 km de lado (Agut, 2012), cuatro de ellas en el municipio de Vitoria-Gasteiz y una en Armiñón. Se localiza ahora en la parte alavesa del río Ebro.

Barlia robertiana (Loisel.) Greuter

BIZKAIA: 30TVN8891, Sopuerta, 106 m, borde de carretera; 30TVN9397, Abanto y Ciérvana-Abanto Zierbena, La Retuerta, 100 m., borde de carretera, 20.02.2012; 30TVN9398, Zierbena, El Tejeñal, 110 m, borde de carretera, 24.02.2015, E.J. Díaz.

Las dos primeras poblaciones fueron cartografiadas por miembros de la Sociedad de Ciencias Naturales de Sestao en 2012 gracias a indicaciones de Manu Océn y Nino Fernández. La población de Sopuerta fue localizada por el micólogo Javier Fernández Vicente y la de Abanto Zierbena por Nino Fernández, Roberto y Jon de la Sociedad Micológica Bihotz Gaztea de Santurtzi, en 2010. Se trata de citas de elevada importancia por tratarse de nuevas poblaciones para Bizkaia, que hasta ahora contaba únicamente con la de la playa de La Arena. Actualmente estas dos poblaciones han sufrido una importante merma al haber sido arrancados todos los especímenes más desarrollados. Esta orquídea está incluida en la Lista Roja de la Flora Vascular de la CAPV bajo el epígrafe "En peligro crítico de extinción" (CR).

\section{Bergenia crassifolia L.}

BIZKAIA: 30TWN0291, Bilbao, Olabeaga, 4 m, en cunetas y ambientes ruderales sobre la Ría del Nervión, 08.02.2014, J. Elorza.

Procedente de Siberia y Asia Central y acostumbrada a climas extremos, posee fuertes rizomas que propician un gran desarrollo de sus poblaciones. Es utilizada en jardinería y para controlar sus poblaciones en muchos casos los rizomas son arrancados y arrojados en zonas cercanas donde arraigan con facilidad.

\section{Botrychium lunaria (L.) Sw.}

BIZKAIA: WN1567 y WN1568, Orozko, Itxina, Lexardi, Altipitatx, repisas herbosas y pastos de diente en zona kárstica, 1144 m, 27.06.2013, S. Patino; 30TWN1965, Zeanuri, Agiñeta, $1050 \mathrm{~m}$, se localizan 10 ejemplares en pastos de diente cercanos al sendero que sube a Gorbea. 
Se aportan tres nuevas localidades para esta especie incluida en la Lista Roja de la Flora Vascular de la CAPV bajo el epígrafe "Casi amenazada" (NT), cuyas únicas poblaciones vizcaínas se encuentran en el macizo del Gorbea y donde quizá sea más abundante de lo que las citas ponen de manifiesto ya que pasa fácilmente inadvertida debido a su pequeño tamaño. De Itxina había sido citada del monte Aitzkorrigane (Patino et al., 2009-2010) y ahora ha sido localizada en otro lugar de este macizo kárstico, sobre repisas herbosas entre Lexardi y Altipitatx.

\section{Bromus inermis Leysser}

BIZKAIA: 30TVN9894, Portugalete, Rivas, próximo al río Ballonti, 20 m, en taludes y herbazales junto a vías de comunicación, 10.06.2014, S. Patino \& J. Valencia.

Planta introducida, subespontánea en ambientes ruderales y en ocasiones cultivada. Hasta el momento contaba con una única cita en la provincia "Mendata, de Albiz a Mamiz, en talud sobre carretera" (Campos et al., 2002). Taxón escaso en la CAPV, con otras dos únicas referencias para Álava ((Aseginolaza et al. 1984; Campos et al., 2002).

\section{Calendula arvensis L.}

BIZKAIA: 30TWN0093, Barakaldo, Zuloko lbarreta, 10 m, sobre terrenos degradados con rellenos calizos, 13.05.2013, S. Patino.

Planta que aparece en pastos secos, tomillares y baldíos. Frecuente en el sur de la Comunidad Autónoma del País Vasco, sin embargo en Bizkaia contaba únicamente con las citas de Punta Lucero (Patino et al., 2001b) y de Sestao (Patino et al., 1990) de donde desapareció al urbanizarse la zona que ocupaba.

\section{Carex pseudocyperus L.}

BIZKAIA: 30TWP063033, Gatika, Butroe, 3 m, cuneta húmeda de la carretera, 22.05.2015, J. Elorza.

Planta de distribución subcosmopolita, rara en la CAPV, aparece en las orillas de cursos de agua o charcas. Se aporta una nueva localidad para esta especie que cuenta con escasas citas puntuales y dispersas por el territorio de Bizkaia. En Patino et al. (20092010) se cita de Ajangiz y de Getxo (Algorta, Fadura) además de recoger algunas citas de otros autores. (Fig. S3).

\section{Carex riparia Curtis}

BIZKAIA: 30TVN9099, Zierbena, La Arena, 4 m, carrizales y vegetación ribereña junto a la desembocadura del río San Mamés, 26.05.2015, S. Patino \& J. Valencia 
Especie subcosmopolita, asociada a herbazales encharcados, tiene una escasa presencia en la CAPV. Se aporta una nueva cita en el occidente vizcaíno para esta gran cárice de la que sólo se conocían las citas de Forua en Urdaibai (Aseginolaza et al., 1984) y donde también se señalan, sin poderse corroborar, la localidad de Aulesti (Navarro, 1982) y Algorta (Guinea, 1949).

\section{Carex rostrata Stokes}

BIZKAIA: 30TWN2468 y 30TWN2469, Zeanuri, puerto de Barazar, $650 \mathrm{~m}$, bordes de arroyo y trampales, A. Prieto; 30TVN6876 y 30 TVN6877 Karrantza Harana/Valle de Carranza, Montes de Ordunte, Llana de Salduero, 1040-1065 m. terrenos encharcados y orillas de charcas sobre suelos higroturbosos, A. Prieto, E.J. Díaz \& S. Patino.

Citada en Aseginolaza et al. (1984) de los Montes de Ordunte y Puerto de Barazar, aportamos cuatro nuevas cuadrículas. Se trata de un taxón muy raro que además está incluido en la Lista Roja de la Flora Vascular de la CAPV como "En peligro de extinción" (EN).

\section{Cephalanthera longifolia (L.) Fritsch}

BIZKAIA: 30TVN9772, Karrantza Harana/Valle de Carranza, Los Jorrios, Ruta del Alimoche pr. Cabaña Enolo, 450 m, claros de encinar cantábrico, 23.05.2012, E.J. Díaz, S. Patino \& F.J. Martínez.

Orquídea rara, de presencia en claros forestales, setos y herbazales. Cuenta con escasas citas en el territorio de Bizkaia. En Aseginolaza et al. (1984) se cita de Mañaria y Arrasate-Mondragon (Udala), mientras que Onaindia (1986) la señala de Zalama. Aportamos una nueva cita.

Cephalanthera rubra (L.) Rich.

ÁLAVA: 30TVN977, Amurrio, Tertanga, 389-582 m, claros de encinar, 12.06.2013, J. Elorza.

Orquídea de distribución submediterránea, presente en hayedos y carrascales pedregosos. Más abundante en la mitad sur de la CAPV. Al norte de la divisoria de aguas solamente se conocía de Trucios/Turtzioz (Prieto, 2000).

Chamaesyce peplis (L.) Prokh.

BIZKAIA: 30TVP9903, Getxo y Sopela, playa Barinatxe, 10 m, dunas 19.07.2012, A. Prieto. 
Se localiza una importante población de este taxón en la playa gracias a indicaciones de Eduardo Renovales. Aunque en 2013 se pensó que la población había desaparecido, se ha vuelto a ver en 2014 y 2015, aunque con menos ejemplares que el primer año.

Habiendo sido citado de hasta diez puntos distintos de la costa vizcaína por diversos autores, a día de hoy solamente se tiene certeza de su presencia en esta localidad. Está incluido en la categoría "En peligro crítico de extinción" (CR) de la Lista Roja de Flora Vascular de la CAPV. (Fig. S4).

\section{Cochlearia aestuaria (Lloyd) Heywood}

BIZKAIA: 30TVN9993, Barakaldo, río Galindo; 30TWN0192 y $30 T W N 0292$ Burtzeña, río Cadagua, 10.04.2011, J. Valencia; 30TWN0194, 30TVN9994, y 30TWN0094, Sestao, río Galindo, 4 m, sobre retazos de marisma en las orillas del río formando grandes manchas, 16.04.2011, S. Patino \& J. Valencia; 30TWP0603, Gatika, Butroe, 4 m, margen de la ría de Butroe, 27.03.2014, J. Elorza \& S. Patino; 30TWN4596, Ondarroa, 1 m, marisma en borde de carrizal, 24.03.2014, A. Prieto; 30TWN2799, Gautegiz Arteaga, Urdaibai, marisma, 0 m, 06.06.2014, A. Prieto; 30TWN2798, Kortezubi, Urdaibai, $4 \mathrm{~m}$, marisma en borde de carrizal, 24.03.2014, A. Prieto.

Endemismo del Golfo de Bizkaia, está presente en zonas fangosas y salobres del litoral. Se aportan diversas citas para la zona del Gran Bilbao donde forma poblaciones de grandes dimensiones en algunos afluentes del río Nervión y se añaden nuevas cuadrículas en la Reserva de la Biosfera de Urdaibai, así como una nueva localidad en las marismas del río Artibai. Su implantación y expansión por el Gran Bilbao parece estar relacionada con la depuración y aumento de la calidad del agua en las últimas décadas.

En Aseginolaza et al. (1984) se cita de Plentzia, y para la zona de Urdaibai en Patino et al. (1991) se señala de Gernika-Lumo, 30TWP20, y Silvan \& Campos (2002) de la cuadrícula 30TWP2501. Está considerado como especie "Vulnerable" (VU) en la Lista Roja de Flora Vascular de la CAPV.

\section{Cochlearia danica L.}

BIZKAIA:30TWP1408, 30TWP1208, Bakio, 30 m, acantilados, 07.04.2015, A. Prieto; 30TWP0409, Gorliz, 35 m, acantilados, 10.4.2015, A. Prieto

Rareza de distribución litoral, presente en áreas bajo la influencia marítima como acantilados costeros. En Aseginolaza et al. (1984) se cita ya de tres puntos de Bizkaia: Bakio, Matxitxako y Laga. Posteriormente fue citada de Zarautz (Lizaur \& Salaverria, 1987). Considerado como "Casi amenazada" (NT) en la Lista Roja de Flora Vascular de la CAPV, estas nuevas citas ayudan a perfilar su distribución en la costa vizcaína. 


\section{Convolvulus lineatus L.}

BIZKAIA: 30TVN9399, Zierbena, Punta Lucero, $156 \mathrm{~m}$, pastos pedregosos y terrenos erosionados con sustrato calizo, 12.09.2013, S. Patino \& E.J. Díaz; 30TVP9800, Getxo, $54 \mathrm{~m}$, pastos pedregosos sobre el acantilado, 11.06.2012, J. Valencia; 30TVP9702, Ibídem, 64 m, pastos pedregosos sobre el acantilado, 15.06.2015, A. Prieto.

Planta de claros y pastos xerófilos o taludes arcillosos calizos, es escasa en el sur y muy rara en los valles atlánticos de la CAPV. Aseginolaza et al. (1984) la citan de diversos puntos del sur de Araba, siendo las primeras citas para Bizkaia las de Plentzia (Alejandre et al., 1987) y de Punta Lucero (Patino \& Valencia, 1989). Se aportan varias cuadrículas para este taxón catalogado como "Con menor riesgo de extinción" (LC) para la CAPV, y rara en el territorio de Bizkaia.

\section{Coronilla glauca L.}

BIZKAIA: 30TWN1883, Lemoa, 70 m, talud de la carretera general, 15.05.2012, J. Elorza.

Cultivada como ornamental, aparece asilvestrada formando una vistosa población sobre resaltes calizos junto a la carretera. Hasta el momento no se tenía conocimiento de su presencia en Bizkaia.

Cotula australis (Sieber ex Sprengel) Hooker fil.

BIZKAIA: 30TWN0590, Bilbao, 2 m, grietas del pavimento en la orilla del Nervión junto al puente de Calatrava, 12.09.2011, S. Patino; 30TWP0405, Plentzia, 4 m, aceras y bordes de jardineras en las calles del pueblo, 24.01.2016, E. Miguel, J. Elorza, B. Zorrakin, J. Valencia \& S. Patino.

Se aportan nuevas localidades para esta especie alóctona, probablemente introducida con semillas utilizadas en trabajos de jardinería y que se adapta con facilidad a los medios urbanos. Las primeras citas se dan en Bilbao y Donostia (Aizpuru et al., 1998). En los últimos años se ha observado un aumento de su presencia en parques y jardines del entorno del Gran Bilbao, con floraciones repartidas a lo largo de todo el año en condiciones de elevada humedad. En Patino \& Valencia (2000) se señalan un par de localidades vizcaínas, y posteriormente en Patino et al. (2009-2010) se citan diversas localidades para Bizkaia así como un par de ellas en territorio de Gipuzkoa. De Cantabria ha sido citada de Santillana del Mar y Santander (Aedo et al., 2000; Durán, 2014). 


\section{Cyclospermum leptophyllum (Pers.) Sprague}

BIZKAIA: 30TVN9897, Getxo, avenida Zugazarte, $6 \mathrm{~m}$, bordes de jardines y grietas de aceras en ambientes sombríos y nitrogenados, 01.09.2010, J. Valencia; 30TVN99408/99935, Getxo, plaza Villamonte de Algorta, $36 \mathrm{~m}$, sobre terrenos ajardinados, 01.10.2012, J. Elorza; 30TVN9992, Barakaldo, Kariga, 8 m, en zonas ajardinadas, 10.10.2013, S. Patino.

Especie anual desconocida hasta ahora en nuestra zona, aparece al igual que otras alóctonas sobre zonas ajardinadas irrigadas, donde prolifera con facilidad. En la Península Ibérica ha sido citada como naturalizada de Valencia y Barcelona, así como de algunas provincias portuguesas. De Bizkaia: Erandio, Lutxana, ha sido repartida por $J$. A. Campos (XX Exsiccata de flora selecta de la AHIM, Centuria 2015).

Dittrichia graveolens (L.) Greuter

BIZKAIA: 30TVN8991, Galdames, El Cerco, 125 m, terrenos alterados junto a antigua vía de Galdames, 04.10.2014, J. Valencia.

Planta desconocida hasta el momento en Bizkaia, y de la que las únicas referencias para nuestra zona son las aportadas por Patino et al. (2009-2010) donde se señala de Murgia así como de la localidad riojana de Conchas de Haro. Citada antaño para Bizkaia como Inula graveolens. Además, existen varias citas para Pamplona (Navarra) (Aizpuru et al., 1996; Lorda 2013) y un pliego de herbario para Cabredo (Navarra) (ARAN 49111) (J. Garmendia, com. pers.).

\section{Dryopteris aemula (Aiton) Kuntze}

BIZKAIA: 30TVN6778, Karrantza Harana/Valle de Carranza, Ordunte, bordes de arroyos en barrancos silíceos, 885 m, A. Prieto, 30TVN6879, Ibídem, 500 m, A. Prieto; 30TVN7080, Ibídem, $400 \mathrm{~m}$, A. Prieto; 30TVN7179, Ibídem, $410 \mathrm{~m}$, A. Prieto; 30TVN7280, Ibídem, 400-535 m, A. Prieto; 30TVN7483, Ibídem, 330 m, A. Prieto; 30TVN7582, Ibídem, $360 \mathrm{~m}$, A. Prieto; 30TVN7584, Ibídem, 425 m, A. Prieto; 30TVN7682, Ibídem, 535 m, A. Prieto; 30TVN7379, Ibídem, Balgerri, Ordunte, 1076 $\mathrm{m}$, rezumaderos en cabecera de arroyo, 28.07.2004, S. Patino \& M. Macias; 30TVN7393, Trucios-Turtzioz, barranco del Remendón, 300 m, cabecera de regato, en margen izquierda, 08.03.2011, S. Patino \& E.J. Díaz; 30TVN7983, Artzentales, Kolitza, 860 m, repisas y pies de cantil silíceo en zona boscosa y húmeda, 06.05.2004, S. Patino \& J. Valencia; 30TVN8491, 30TVN8492, Sopuerta, Tresmoral, 210-229 m, fondo de barranco silíceo, S. Patino \& E.J. Díaz; 30TVN8691, Ibídem, Ortega, 235 m, talud de pista, en zona sombría y fresca, 14.12.2013, S. Patino; 30TVN8690, Sopuerta, barrio La Cilla, $124 \mathrm{~m}$, base de talud de areniscas en zona húmeda y sombría, 
23.05.2012, E.J. Díaz \& S. Patino; 30TVN9786, Güeñes, La Cuadra, 199 m, fondo de barranco silíceo, 07.01.2014, E.J. Díaz \& S. Patino; 30TWP1504, Bakio, Emerando erreka, 84 m, bosque de ribera, 30.12.2013, S. Patino; 30TWP1607, Ibídem; 30TVN9292, Galdames, Montes de Triano pr. Pico el Gallo, 540 m, rezumaderos y bordes de regato en fondo de barranco sombrío y húmedo, 25.03.2006, E.J. Díaz \& S. Patino; 30TWP1802, Meñaka, Sollube, 450-460, 500 m, A. Prieto, S. Patino \& J. Valencia; 30TWP1804, Bermeo, Sollube, 230 m, A. Prieto, S. Patino \& J. Valencia; 30TWP2104, Bermeo, Almike, barranco de Artigas, $130 \mathrm{~m}$, fondo de barranco, 03.06.2001, A. Prieto, S. Patino \& J. Valencia; 30TWN2381, Amorebieta-Etxano, barrio Bernagoitia, Arroyo de Areotzerrota, 400 m, en barranco encajado junto a cascada, 19.07.2007, A. Prieto \& S. Patino; 30TWP1805, Mungia, Infernu erreka, 170 m; 30TWN2368, Zeanuri, Barazar, 640 m; 30TVN9292, Trapagaran, La Arboleda, 574 m, fondo de barranco silíceo, 10.06.2011, S. Patino \& E.J. Díaz.

Helecho de bosques sombríos y barrancos silíceos con fuerte influencia cantábrica. Se aportan diversas localidades vizcaínas que, junto a las ya conocidas, ayudarán a perfilar el área de este taxón incluido en la categoría "Casi amenazada" (NT) en la Lista Roja de la Flora Vascular de la CAPV.

\section{Epipactis helleborine subsp. helleborine (L.) Crantz}

BIZKAIA: 30TVN7391, Karrantza Harana/Valle de Carranza, Sopeña, 515 m, encinar sobre calizas 20.07.2015, S. González Ahedo.

Orquídea escasa en la CAPV, de ambientes forestales. Desconocida hasta ahora en el occidente vizcaíno, la nueva población cuenta con más de una docena de ejemplares que llegan a madurar sin problemas.

Hasta el momento, la cita de Mañaria (Aseginolaza et al., 1984) y la de Oba (Navarro, 1982) eran las únicas referencias para el territorio de Bizkaia. Se conocen citas dispersas para el norte de Burgos (Alejandre et al., 2006) y Cantabria (Durán, 2014).

Epipactis kleinii M.B. Crespo, M.R. Lowe \& Piera].

BIZKAIA: 30TVN7391, Karrantza Harana/Valle de Carranza, Sopeña, 500 m, encinar sobre calizas, 20.07.2015, S. González Ahedo.

Endemismo ibérico, presente en el sur del territorio y de manera escasa, aparece en pastos y claros forestales pedregosos calizos. En el territorio de Bizkaia se le conocía únicamente de las localidades de Gorliz y Duranguesado, esta última en Monte Mugarra (Aseginolaza et al., 1984), por lo que la que aquí se reseña tiene un interés corológico importante para Bizkaia. 


\section{Epipactis palustris (L.) Crantz}

BIZKAIA: 30TVN7191, Karrantza Harana/Valle de Carranza, Ranero, 585 m, terrenos higroturbosos, 11.7.2014, A. Prieto.

Orquídea con presencia escasa en el tercio central de la CAPV y muy rara en el litoral y valles atlánticos, aparece en prados húmedos y trampales calizos. Única cita en firme para Bizkaia de esta especie que contaba con la referencia de Bilbao (Gandoger, 1917), cita que no ha podido ser corroborada debido a la transformación de la zona en el último siglo. Localizados varios ejemplares en un pequeño humedal. Considerada como "Vulnerable" (VU) en la Lista Roja de la Flora Vascular de la CAPV.

\section{Galega officinalis L.}

BIZKAIA: 30TWN0291, Bilbao, Zorrotza, 7 m, taludes, 18.10.2013, J. Valencia \& J. Elorza; 30TVN9894, Portugalete, Rivas, 20 m, taludes y descampados cercanos a la autovía, 20.05.2013, J. Valencia \& S. Patino.

Aparece en taludes y zonas ajardinadas formando en algún caso poblaciones de cierta entidad. Su origen en la zona pudiera estar ligado a las siembras que se realizan esporádicamente con fines ornamentales. Desconocida hasta la fecha en el territorio de Bizkaia y muy rara en la CAPV, donde se la conoce únicamente del sur de Álava, y en el norte de Navarra. Para Cantabria Sánchez y Valdeolivas (1995) recogen la referencia de Camargo (cf. Durán, 2014).

\section{Genista pilosa L.}

GIPUZKOA: 30TWN3656, Leintz-Gatxaga, Usokoaitza, 820 m, claros de enebral-brezal, 14.05.2014, S. Patino.

Aparece en brezales de suelos silíceos, escasa en las montañas de transición y rara en valles atlánticos. En Gipuzkoa solamente se conoce del monte Jaizkibel. Por otro lado, Aseginolaza et al. (1984) aportan diversas localidades alavesas alguna de ellas próxima a la aquí citada.

\section{Glaucium flavum Crantz}

BIZKAIA: 30TVN9099, Zierbena, La Arena, 10 m, dunas de la playa, 10.04.2013, J. Valencia.

Papaverácea de arenales costeros o de baldíos removidos del interior. Especie dada por extinguida en la CAPV y que nuevamente aparece de forma sorprendente en la zona. Quizá su aparición está ligada a la erradicación accidental de Carpobrotus debido a las últimas galernas y por la gran capacidad de germinación de sus semillas que han podido permanecer sepultadas por esa especie invasora. (Fig. S5). 


\section{Glaux maritima L.}

BIZKAIA: 30TWN4596, Ondarroa, 1 m, marismas, 25.04.2014, A. Prieto.

Citada para la provincia de Gernika y Lekeitio (Aseginolaza et al., 1984) donde también se recoge una antigua referencia para Portugalete (Wilkomm \& Lange, 1870).

Su hábitat son fangos salobres de juncales de Juncus maritimus de marismas litorales. Nueva cita que ayuda a perfilar el área de esta primulácea, escasa en nuestro litoral. (Fig. S6).

\section{Himantoglossum hircinum (L.) Spreng}

BIZKAIA: 30TWN1491, Lezama, $67 \mathrm{~m}$, un ejemplar en borde de carretera, 26.05.2014, Ricardo García; 30TWP0407, Gorliz, ha sido localizada en las dunas de Astondo por indicación de Daniel García.

Planta de pastos y ribazos, con presencia desde dunas litorales hasta montes de mil metros. Se aportan dos nuevas citas para esta vistosa orquídea, cuya primera cita para Bizkaia se debe a K. Amaia y A. der Kinderen que localizaron un ejemplar en Getxo (Llorente et al., 2009). Existen referencias de otro ejemplar en Zierbena, en el barrio de La Cuesta (Manu Océn, com. pers.). Se trata de una cita de importancia corológica para Bizkaia, aunque queremos destacar que, al igual que otras orquidáceas, parece estar en expansión. Esta Orquídea está incluida en la Lista Roja de la Flora Vascular de la CAPV con la categoría de "Casi amenazada" (NT).

Huperzia selago (L.) Bernh. ex Schrank et Mart.

BIZKAIA: WN1266, Orozko, sobre Algorta, 933 m, talud pedregoso con brezal argomal, junto a la pista a Austigarmin, 27.06.2013, S. Patino; 30TWN190, Zeanuri, Agiñeta, 655-1050 m, pastos de diente junto al camino a Gorbea, 12.05.2015, S. Patino.

Pteridófito que aparece en ambientes húmedos de brezales o repisas tanto silíceas como calizas lavadas. Aunque ha sido citada de localidades cercanas, incluimos éstas por tratarse de una especie catalogada como "Casi amenazada" (NT) (Fig. S7).

\section{Isopyrum thalictroides $\mathrm{L}$.}

BIZKAIA: 30TWN2578, Durango, Aramotz, Parque Natural de Urkiola; 30TWN2578, Ibídem, 663 m; 30TWN2577, Ibídem, 786 m; 30TWN2478, Ibídem, 764-768 m, hayedos sobre calizas con suelos profundos ricos en humus, 15.04.2015, J. Elorza, S. Patino \& J. Valencia. 
Ranunculácea que aparece sobre el mantillo de hayedos, robledales, fresnedas y alisedas, preferiblemente del piso colino (mesotemplado) atlántico. Especie escasa que hasta el momento contaba con un par de citas provinciales. Aseginolaza et al. (1984) la citaron de Orozko y Sierra de Gorbea, y en Patino \& Valencia (1989) se cita de la parte cántabra de las Peñas de Ranero, a escasos metros del límite con Bizkaia, localidades bastante alejadas de la que ahora se aporta y donde forma manchas de considerable extensión.

Juncus acutus subsp. acutus L.

BIZKAIA: 30TWP0408, Gorliz, 120 m, talud rocoso junto a puente, 04-10-2014, J. Valencia; 30TWP1108, Lemoiz, 50 m, pequeña zona húmeda sobre acantilado, 25.03.2015, A. Prieto; 30TWN0099, Leioa, Lamiako, 4 m, sobre terrenos rellenados de antigua marisma y márgenes del río Gobelas en su desembocadura a la ría del Nervión, B. Zorrakin.

Se aportan dos nuevas localidades vizcaínas para esta especie catalogada "Casi amenazada" (NT). Resaltar la cita de Lamiako donde vive en dos manchas diferenciadas, una junto al río Gobelas y otra sobre rellenos en compañía de otras especies marismeñas, contabilizando varias docenas de macollas.

\section{Leycesteria formosa Wall.}

BIZKAIA: 30TWN0388, Bilbao, Recalde-Arraiz, $125 \mathrm{~m}$, naturalizada en repisas de roquedos calizos, junto al talud de la carretera, S. Patino \& R. Toral, 13.06.2010.

Especie originaria del Himalaya, usada en jardinería por sus vistosas flores y que en algunos casos se asilvestra en los alrededores.

\section{Lilium martagon L.}

BIZKAIA: 30TWN2578, Durango, Aramotz, Parque Natural de Urkiola, 663 m, ladera orientada al norte, sobre repisas frescas ricas con suelos ricos en humus en ambiente de hayedo, 15.04.2015, J. Elorza, S. Patino \& J. Valencia.

Al igual que su congénere $L$. pyrenaicum, vive en nuestro territorio ocupando un amplio gradiente altitudinal, aunque es bastante más escaso. La población que aquí se cita agrupa a varias decenas de ejemplares. Nueva cita para esta especie que cuenta con escasas localidades dispersas en la provincia de Bizkaia. 


\section{Lythrum tribracteatum Spreng.}

ARABA: 30TWN2911, Navaridas, 540 m, borde de laguna salada, 31.7.2015, A. Prieto.

Género ligado a la presencia de agua, la especie aparece en juncales y herbazales salobres. Se trata de la segunda cita para la CAPV, siendo la primera la de Aizpuru et al. (1996) de la laguna de Musco, en Laguardia. Los mismos autores la citan de Navarra en Viana, laguna de Las Cañas; en Pitillas; y en Urraul Bajo, Artieda.

\section{Lycopodium clavatum L.}

BIZKAIA: 30TVN7781, Karrantza Harana/Valle de Carranza, Ordunte, monte Burgueño, 1000 m, brezales de la cara norte 19.07.2013, A. Prieto.

Única especie del género, con óptimo en brezales silíceos atlánticos del cinturón colino. Conocida del monte Gorbea (Aseginolaza et al., 1984), esta cita se suma a la de Ordunte (Pico Balgerri) que fue señalada en Patino et al. (2009-2010), donde además se citaban también algunas localidades conocidas del norte burgalés. Así mismo, ha sido localizada en la zona de Salduero y Monte Grande (Joseba Garmendia, comunicación personal). Aparece ahora en el extremo oriental de la Sierra de Ordunte en un ambiente muy similar al de Balgerri.

Especie incluida en la Lista Roja de la Flora Vascular de la CAPV en la categoría "Casi amenazada" (NT). (Fig. S8).

\section{Moricandia arvensis (L.) DC.}

BIZKAIA: 30TVN9099, Muskiz, playa de La Arena, 4 m, en terrenos degradados y escombreras, 20.03.2011, S. Patino.

Especie ruderal con gran poder de colonización que suele formar vistosas poblaciones debido a sus flores rosas al comienzo de la primavera. Para Bizkaia ha sido citada únicamente del barrio bilbaíno de Otxarkoaga en Barredo Pérez (2005). Segunda cita provincial para esta especie alóctona cada vez más abundante en nuestra zona.

\section{Narcissus bulbocodium L.}

BIZKAIA: 30TVN9791, Barakaldo, Argalario, 480 m, pastos de diente, sustrato silíceo, 29.4.2011, S. Patino; 30TVN0089, Barakaldo, Arroletza, 350 m, pastos en sustrato silíceo, 17.03.2005, J. Valencia; 30TVN9889, Barakaldo, Peñas Blancas, 350 m, pastos pedregosos calizos, S. Patino \& J. Valencia; 30TVN9593, Valle de Trápaga-Trapagaran, la Reineta, 365 m, Pastos de diente junto a la carretera, 15.03. 2007, S. Patino; 30TVN7279 y 30TVN7279, Karrantza Harana/Valle de Carranza, Balgerri, 877 m y 
$1047 \mathrm{~m}$, brezales de la cara norte, 29.03.2008, S. Patino; 30TVN7491 y 30TVN7492, Ibídem, Armañón, Los Jorrios, 710 m y 747 m, claros de brezal, en cerro con afloramientos calizos, 18.03.2015, J. Elorza, S. Patino, J. Valencia \& B. Zorrakin; 30TVN6777, 30TVN6876, 30TVN6877, 30TVN6977, Ibídem, Ordunte, Salduero, 1030-1080 m, brezales y claros de brezal con sustrato silíceo, A. Prieto, J. Elorza, E.J. Díaz, E. Miguel, \& S. Patino; 30TVN6788, Ibídem, pr. el Mazo, $425 \mathrm{~m}$, pastos con afloramientos calizos, 17.03.2005, E. Miguel \& S. Patino; 30TVN9194, Abanto y Ciérvana-Abanto Zierbena, Cortes, 350 m, herbazales y claros de brezal, 03.03.2008, E.J. Díaz; 30TVN0283, Alonsotegi, Ganekogorta, 740 m, ladera silícea, S. Patino, R. Toral \& J. Valencia; 30TWN2182, Amorebieta-Etxano, Arroyo Leginetxe, claros de brezal sobre el regato, 09.02.2006, E. Prieto \& S. Patino; 30TWN0890 y 30TWN0990, Bilbao, pr. Monte Abril, pastizales y claros de matorral, 04.04.2007, J. Valencia; 30TVN9292, 30TVN9390, 30TVN9391, Galdames, Montes de Triano, 620-650 m, pastos pedregosos y claros de brezal, 25.03.2005, S. Patino \& J. Valencia; 30TVN9487 y 30TVN9488, Ibídem, Tillitu, 600 y 750 m, pastizales y claros de brezal, 25.03.2005, S. Patino \& J. Valencia; 30TWN1189, Galdakao, 410 m, pastizales silíceos, J. Valencia; 30TVN9586, Güeñes, Eretza, $750 \mathrm{~m}$, pastizales junto al sendero al Eretza, 25.03.2005, S. Patino \& J. Valencia; 30TWP0709, Lemoiz, $150 \mathrm{~m}$, claros de brezales y argomales cercanos a los acantilados, 08.03.2007, A. Prieto; 30TWN1190 y 30TWN1290, Lezama, 275 m, ladera silícea, A, Prieto; 30TWN1803, Meñaka, Sollube, $350 \mathrm{~m}$, A. Prieto; 30 TWN0990 y 30TWN1091, Zamudio,120 m, pastos con sustrato silíceo, 20.03.2012, J. Valencia.

Citado para la provincia de Karrantza Harana/Nalle de Carranza, Serantes, UrduñaOrduña, Sierra de Gorbea y Oiz (Aseginolaza et al., 1984), Bariego \& Gastón (2002) lo citan de Balmaseda y EKOS (2005) de Urdaibai.

Se aportan diversas citas, algunas de ellas incluidas en la "Cartografía de especies amenazadas de Bizkaia" (Patino et al. 2013), con las que se trata de actualizar su distribución en la provincia. Este taxón se corresponde con lo que en Aizpuru et al. (1999) se determina como Narcissus bulbocodium subsp. citrinus (Baker) Fern. Casas, y está incluido como tal en la categoría "Con menor riesgo de extinción" (LC) de la Lista Roja de Flora Vascular de la CAPV.

\section{Narcissus minor subsp. minor L.}

BIZKAIA: 30TVN9393, Abanto y Ciérvana-Abanto Zierbena, las Calizas, 403 m, pastos pedregosos calizos, S. Patino \& J. Valencia; 30TVN9292, Galdames, El Sauco, Peña San Juan, $640 \mathrm{~m}$, pastos de diente, sustrato calizo, 06.04.2005, S. Patino; 30TVN9389 y 30TVN9390, Galdames, Guiuruz, 600-650 m, pastos pedregosos calizos, 10.4.2007, E.J. Díaz, S. Patino \& J. Valencia; 30TVN9487, Galdames, La Berenilla, 575 m, pastos sobre calizas, 10.04.2007, E.J. Díaz, S. Patino \& J. Valencia; 30TVN9489, Galdames, Pico la Cruz, 740 m, 10.04.2007, E.J. Díaz, S. Patino \& 
J. Valencia; 30TWN0485,Bilbao, Pagasarri, 650 m, repisas y grietas de lapiaz, cercanas a la cumbre, 16.02.2008, E. Miguel, J. Valencia, R. Toral \& S. Patino; 30TWN1469, Orozko, Itxina, pr. Atxular, 17.03.2007, 1025 m, repisas herbosas y grietas de lapiaz, S. Patino \& J. Valencia; 30TWN1666, Orozko, Gatzarrieta, 1140 m, pastos pedregosos calizos, 17.03.2007, S. Patino \& J. Valencia; 30TWN1567, Orozko, Gorosteta, 1230 m, repisas y grietas de lapiaz, 17.03.2007, S. Patino; 30TWN1466, Orozko, Ibarra, Austiarmin, 1060 m, repisas y grietas de lapiaz, 01.03.2007, S. Patino; 30TWN1766, Zeanuri, Gatzarrieta, 1090 m, pastos pedregosos calizos, 17.03.2007, S. Patino \& J. Valencia; 30TWN2066 y 30TWN2065, Zeanuri, Arimekorta, 910-925 m, pastos de diente sobre calizas, 10.03.2007, S. Patino \& J. Valencia; 30TWN2377, Dima, Artaungane, $732 \mathrm{~m}$, sobre pastos pedregosos calizos ocupando una superficie considerable, 05.04.2010, S. Patino; 30TWN2181, Dima, Aramotz, 485 m, pr. Belatxikieta, E. Miguel \& S. Patino; 30TWN2477, Urkiola, Leungane, 950 m, rellanos y repisas herbosas sobre calizas, 15.04.2008, S. Patino \& J. Valencia, Trucios-Turtzioz, Los Jorrios, 30TVN7592, 760 m, terrenos pedregosos calizos, 15.02.2011, E.J. Díaz, S. Patino \& F.J. Martínez.

Para el territorio de Bizkaia Pérez de Ana (2003) lo cita de Galdames (Peña Pastores) y Aseginolaza et al. (1984) lo señalan de Gorbea (Arrabakoate) y Urduña-Orduña, como N. minor.

Se aportan diversas citas, algunas de ellas incluidas en la "Cartografía de especies amenazadas de Bizkaia" (Patino et al., 2013) con las que se trata de actualizar el conocimiento que se tiene de esta especie en la provincia. Este taxón se corresponde con lo que en Aizpuru et al. (1999) se determina como Narcissus asturiensis (Jordan) Pugsley subsp. jacetanus (Fern. Casas) Uribe-Echebarría, y está incluído como tal en la categoría "Con menor riesgo de extinción" (LC) de la Lista Roja de la Flora Vascular de la CAPV.

\section{Narcissus pseudonarcissus subsp. pseudonarcissus L.}

BIZKAIA:30TVN7491, Karrantza HaranaNalle de Carranza, Armañón, 710 m, brezales y bordes de hayedo 04.04.2015, A. Prieto.

Narciso de prados húmedos silíceos y de hayedos acidófilos (Aizpuru et al., 1999). En el País Vasco está presente en varias localidades de Araba y Bizkaia, en este último territorio histórico se han citado poblaciones en 15 cuadrículas UTM de $1 \times 1 \mathrm{~km}^{2}$, la mayoría de ellas ubicadas en Ordunte (Patino et al., 2013). Aportamos cita en el Parque Natural de Armañón.

Este taxón se corresponde con lo que en Aizpuru et al. (1999) se determina como Narcissus nobilis (Haw.) Schultes fil., y está incluido como "Casi amenazada" (NT) en la Lista Roja de la Flora Vascular de la CAPV. 


\section{Ophioglossum lusitanicum L.}

BIZKAIA: VN9684, Güeñes, Sodupe, 111-139 m, rellanos y repisas de ladera margosa a la izquierda del desfiladero, 03.02.2011, S. Patino; VN9887, Güeñes, Zaramillo, 115$145 \mathrm{~m}$, en claros de encinar sobre el pueblo de Zaramillo junto a unas torres de alta tensión, 27.01.2010, S. Patino.

CANTABRIA: 30TVN7205, Liendo, Monte Candina, $300 \mathrm{~m}$, en pastizal soleado junto al camino a la cumbre, zona con pequeñas escombreras mineras, 09.02.2011, S. Patino.

Pequeño helecho que vive sobre pastos musgosos, ya conocido tanto de la costa cántabra como del "Corredor del Cadagua". Se aportan dos nuevas localidades, una cántabra y otra vizcaína, para perfilar el área de esta especie protegida y escasa en nuestra zona. Incluida en la categoría "Vulnerable" (VU) de la Lista Roja de Flora Vascular de la CAPV. (Fig. S9).

\section{Ophioglossum vulgatum L.}

BIZKAIA:30TWN0485, Bilbao, Pagasarri, 647 m, depresión con pasto de diente junto al refugio, 14.05.2010, E. Miguel, S. Patino \& J. Valencia; 30TWN2578, Durango,Aramotz, 730 m, 30TWN2577, Ibídem, 786 m, sobre pastos frescos en zonasligeramente deprimidas, 15.04.2015, J. Elorza, S. Patino \& J. Valencia; 30TWN2576, Mañaria, Parque Natural de Urkiola, arroyo Erleabeko, 450 m, 14.06.2013, A. Prieto.

Pequeño helecho que prefiere prados húmedos de alisedas o robledales de fondo de valle. Ya citado en cuadrículas ubicadas al norte del Parque, se citan varias localidades nuevas para este espacio natural. Incluido en la Lista Roja de la Flora Vascular de la CAPV en la categoría "Casi amenazada" (NT).

Ophrys aveyronensis (J.J. Wood) Delforge

ARABA: Amurrio, Sierra Salvada, 30TVN9758, 430.515 m 08.06.2014, E.J. Díaz, A. Prieto \& S. Patino.

Geófito de pastos mesófilos y xerófilos del cinturón colino atlántico, en la parte más occidental del territorio. De una cuadrícula cercana aunque en territorio vizcaíno, Urduña-Orduña, VN9760, 400 m, fue citada como O. archnitiformis en Aseginolaza et al. (1984). Incluido en la Lista Roja de la Flora Vascular de la CAPV en la categoría "Vulnerable" (VU). (Fig. S10). 


\section{Ophrys speculum Link}

BIZKAIA: 30TWP0307, Gorliz, 46 m, pastos pedregosos sobre acantilado, 10.04.2015, A. Prieto.

Aparece en pastos secos y claros de romerales y tomillares de la parte septentrional de la CAPV (Aizpuru et al., 1999). Hasta el momento, la única cita conocida de esta orquídea en el territorio de Bizkaia se debe a Carlón et al. (2010) que la citan del entorno del monte Serantes. La cita que aportamos de esta especie situada en la vertiente cantábrica de la CAPV, cuenta con un número escaso de individuos.

\section{Orchis italica Poir.}

ARABA: 30TWN4629, Arraia-Maeztu, Parque Natural de Izki, 810 m, borde de pista, 21.05.2013, S. Pérez \& A. Prieto.

Aparece en claros de comunidades de coscoja (Quercus coccifera) y de matorrales termófilos (Aizpuru et al., 1999). Por indicaciones de Jonathan Rubines se localizan dos ejemplares de esta singular orquídea en el parque natural de Izki. Se ha citado de varias localidades alavesas de Kuartango (Ruiz Campo, 2013) y de Hueto de Abajo (P. Urrutia, com. pers.). Está considerada como especie "Vulnerable" (VU) en la Lista Roja de la Flora Vascular de la CAPV.

\section{Orchis militaris L.}

BIZKAIA: 30TVN8991, Galdames, Laiseca de Abajo, 70 m, margen de pista, 03.05.2010, B. Zorrakin; 30TWN3186, Munitibar-Arbatzegi Gerrikaitz, Monte Oiz, 780 m, margen de la pista, 18.05.2013, Oskar Telletxea.

Vive en pastos y claros de bosque. Especie muy rara en la vertiente cantábrica del País Vasco donde hasta el momento se conocía solamente una antigua cita de Heintz (Gredilla,1913), referenciada por Aseginolaza et al. (1984) en Eskoriatza, y donde se supone que pudiera tratarse de O. purpurea. Recientemente se ha localizado en Punta Lucero (Ruiz Campo, 2007)

En la vecina comunidad de Cantabria únicamente se conoce de Villaescusa de Ebro, Valderredible, 30TVN34, 800 m, pradera, 28.05.1985, donde fue recolectada por C. Aedo (MA 621471).

\section{Orchis morio L.}

BIZKAIA: 30TWN0485, Bilbao, Pagasarri, $650 \mathrm{~m}$, pastos de diente cercanos al refugio, 25.05.2011, E. Miguel, S. Patino, A. Prieto, J. Valencia \& Rafa Toral. 
Relativamente frecuente al sur de la divisoria de aguas, pero sin referencias anteriores para el territorio de Bizkaia. Novedad provincial.

\section{Orchis papilionacea L.}

CANTABRIA: 30TVP2111, Piélagos, playa de Liencres, 10 m, dunas, 21.4.2013, S. Pérez \& A. Prieto.

Aportamos nueva cita para esta planta rara en Cantabria. Se observan dos ejemplares creciendo en medio de las dunas, en zona pisoteada. Mientras en Navarra cuenta con las citas de Ororbia (Van der Sluys \& González, 1982), Lazagurria, Viana, Mendavia, Liedena y Olague (Lorda López, 2013) y en Bizkaia se conoce de Sondika (Cadiñanos et al., 2010),

en el Catalogo de la Flora Vascular de Cantabria (Durán Gómez, 2014) se recogen tres citas lebaniegas alejadas de la que aquí se aporta, de ahí su interés desde el punto de vista corológico. (Fig. S11).

\section{Physalis peruviana L.}

BIZKAIA: 30TVN9280, Gordexola, El Pontón, 80 m, ambientes ruderalizados, 12.12.2015, J. Elorza.

Especie americana, cultivada por sus frutos para la elaboración de postres y que ocasionalmente se asilvestra en las inmediaciones de zonas urbanas. No se tenía constancia de su presencia en Bizkaia. Willkomm \& Lange (1865) citan de San Sebastián, de donde es reseñado igualmente por Gredilla (1913) y Sennen (1929).

\section{Pinguicula lusitanica L.}

ARABA: 30TVN8841, Valdegovía, Quejo, 720 m, 30TVN8940 y 30TVN8939, Valdegovía, Nograro, 780 - 840 m, encharcamientos en bordes de arroyos, 18.07.2016, A. Prieto.

BIZKAIA:30TVN7379, Karrantza Harana/Nalle de Carranza, Lanzas Agudas, 1040 m, junto a regatas de agua y terrenos encharcados, 29.03.2008, S. Patino; 30TVN7279, Ibídem, Balgerri, 805 m, rezumaderos y zonas húmedas, 29.03.2008, S. Patino; 30TVN6777, Ibídem, Llano de Salduero, 1060 m, turberas y terrenos encharcados, E.J. Díaz, Fran, \& S. Patino, 17.06.2011; 30TVN7191, Ibídem, Santecilla, 573 m, terrenos higroturbosos en ladera sur, 11.07.2014, A. Prieto; 30TVN7391, Ibídem, pr. Sopeña, $710 \mathrm{~m}$, pequeñas zonas húmedas sobre el encinar de Sopeña, S. Patino \& E.J. Díaz; 30TVN9988, Alonsotegi, pr. Barrio Zamundi, 302-315 m, trampales y zonas 
rezumantes, 06.06.2008, E.J. Díaz, O. Orrantia \& S, Patino; 30TVN9987, 30TVN9887 y 30TVN9888, Alonsotegi, pr. Peñas Blancas, 330-340 m, trampales y zonas rezumantes en borde de pista y junto a pequeños arroyos, 06.06.2008, E.J. Díaz \& S. Patino; 30TVN9889, Barakaldo, El Regato, barranco del río Loiola, 80 m, talud rezumante de pista; 30TVN9690 y 30TVN9790, Ibídem, 245 m, talud rocoso y rezumante sobre la pista, 10.05.2007, S. Patino, E. Otxoa \& J. Valencia; 30TVN9989, Barakaldo, Tellitu, camino a Peñas Blancas, 280 m, pequeños manantiales, S. Patino \& J. Valencia; 30TVN9391, Galdames, 630 m, pequeño trampal, 4.8.2014, A. Prieto; 30TWN0485, Bilbao, Pagasarri, 440 m, taludes rezumantes junto a pista, 10.05.2008, E. Miguel, A. Prieto, R. Toral, S. Patino \& J. Valencia; 30TVN7491, Trucios-Turtzioz, Armañón, $550 \mathrm{~m}$, taludes rezumantes junto a pista, 15.05.2007, E. Miguel, A. Prieto, S. Patino \& J. Valencia, 30TVN7293, Trucios-Turtzioz, Violedo, 357-654 m, Trampales, 15.05.2008, E.J. Díaz, I. Aguinaco \& S. Patino; 30TWN0183 y 30TWN0283, Arrankudiaga, Ganekogorta, 690 m, rezumaderos de agua, S. Patino; 30TWN0472, Orozko, Jesuri, 610-640 m, manantiales y rezumaderos, sustrato silíceo, A. Prieto; 30TWN1367, Orozko, humedal de Algorta, 820 m, terrenos encharcados y trampales en fondo de valle, S. Patino, T. Oyanarte, J. Valencia \& J. Elorza; 30TWN2281, Amorebieta-Etxano, pr. Belatxikieta, $480 \mathrm{~m}$, rezumaderos y trampales, S. Patino; 30TWN2289, Muxika, Urdaibai, 400 m, rezumaderos, A. Prieto; 30TWP2204, Busturia, $250 \mathrm{~m}$, pequeños trampales, A. Prieto; y 30TWP2200, Errigoiti, 160 m, rezumaderos en ladera silícea, $A$. Prieto.

Esta pequeña planta pasa fácilmente desapercibida en turberas y manantiales de suelos silíceos de los valles atlánticos. Se aportan nuevas localidades para esta especie incluida en la Lista Roja de la Flora Vascular de la CAPV bajo el epígrafe "Casi amenazada" (NT). (Fig. S12).

\section{Pistacia lentiscus L.}

BIZKAIA: 30TVN9094, Abanto y Ciérvana-Abanto Zierbena, 300 m, ambiente de encinar, 06.12.2013, E.J. Díaz \& S. Patino; 30TVN9098, Muskiz, 14 m, sobre restos de dunas fósiles, 06.12.2013, E.J. Díaz \& S. Patino; 30TVN9198, Ibídem, Montaño, 100 m, entre matorral mediterráneo, 10.12.2013, S. Patino \& J. Valencia; 30TVN9199, 64 m y 30TVP9000, 61 m, Muskiz, Zierbena, La Arena, 06.12.2013, E.J. Díaz \& S. Patino; 30TVP9100, Zierbena, Punta Lucero, 150 m, 06.12.2013, E.J. Díaz \& S. Patino; 30TWP0105 y 30TWP0306, Barrika, 80 m y 40 m, brezales secos atlánticos costeros de Erica vagans y ambiente de encinar, A. Prieto; 30TWP0307, 30TWP0308, 30TWP0406, Gorliz, 31 m, playa y acantilados, dunas fósiles, 21.09.2013, S. Patino, T. Oyanarte, E. Miguel \& J. Valencia; 30TWP0507 y 30TWP0508, Ibídem, 60 m y 160 m, subida hacia el Ermua, A. Prieto; 30TWP0607, Ibídem, hacia Lemoiz, 80 m, A. Prieto; 30TWP0600 y 30TWP0700, Laukiz, de Butroe a Laukiz, 20-60 m, talud de 
carretera, ambiente de encinar, 21.09.2013, S. Patino, T. Oyanarte, E. Miguel \& J. Valencia, 30TWP1710, Bakio, San Juan de Gaztelugatxe, 65 m, acantilados bajo la ermita, 30.12.2013, S. Patino; 30TVN9097, Muskiz, Pico Ramos, 20-195 m, ambiente de encinar y pastos, 30.12.2013, E.J. Díaz.

Este arbusto perennifolio tiene mayor presencia en coscojares y encinares de la vertiente mediterránea aunque también puede aparecer en encinares cantábricos costeros. En este trabajo se aportan numerosas localidades de esta especie catalogada como "Con menor riesgo de extinción" (LC) en la Lista Roja de la Flora Vascular de la CAPV.

\section{Polygonatum verticillatum (L.) All.}

BIZKAIA: 30TVN6688, Karrantza Harana/Valle de Carranza, Peña El Moro, 805 m, en grietas kársticas cercanas a la cumbre, 05.07.2012, S. Patino.

Precisa de grietas de lapiaz con suelos sombríos, ricos en materia orgánica. Planta escasa en la CAPV, del occidente vizcaíno ha sido citado de Karrantza HaranaNalle de Carranza, Zalama, así como del vecino Valle de Soba, ésta en territorio cántabro (Patino et al., 2009-2010), y de la localidad vizcaína de Trucios-Turtzioz (Aseginolaza et al., 1984).

\section{Prunus insititia L.}

BIZKAIA: 30TWP102016, Gatika, 78 m, orla de encinar, 30.08.2015, J. Elorza.

Aparece en setos frescos y alisedas, es también conocido como P. insititia L. (Aizpuru et al., 1999) y se la considera originaria del Cáucaso. Más abundante en Gipuzkoa, donde contaba con diversas localidades (Aseginolaza et al., 1984). Sin embargo, en Bizkaia hasta el momento contaba únicamente con una antigua cita de Allorge (Allorge \& Allorge, 1941) para Lekeitio, recogida por Guinea (1953) en su "Geografía botánica de Santander".

\section{Pteris vittata $\mathrm{L}$.}

BIZKAIA: 30TWN0547, Bilbao, Deusto, 4 m, muro de la ría de Bilbao, J. Elorza

Se trata de un helecho cuyo hábitat son roquedos calizos rezumantes y cursos de agua. Muestra una amplia distribución tropical y subtropical, presente también en territorios mediterráneos y macaronésicos. En la Península Ibérica se distribuye a lo largo de la costa mediterránea, desde Cádiz hasta Barcelona, aunque es más abundante en Andalucía. En el norte peninsular únicamente se conocía de La Coruña, por lo que la cita que se aporta constituye novedad para el País Vasco. (Fig. S13). 


\section{Quercus coccifera L.}

BIZKAIA: 30TVP9100, Zierbena, Punta Lucero, 195 m, roquedos calizos, 04-09-2005, J. Valencia. Una única mata de 2 metros de diámetro.

Arbusto perennifolio de pequeña estatura que forma parte principalmente de la orla de carrascales y quejigares. Distribuido por el tercio meridional de la CAPV, aparece en lugares secos y soleados, sobre suelos pedregosos calizos. Especie catalogada como "Con menor riesgo de extinción" (LC) en la Lista Roja de la Flora Vascular de la CAPV.

\section{Ranunculus amplexicaulis L.}

BIZKAIA: 30TWN187644, Zeanuri, Iginiger, Bedargogor, 1355 m, 12.05.2015, S. Patino.

Planta que vive sobre terrenos pedregosos, aparece en pastos o sobre repisas herbosas de ambiente montano. Aunque conocido de zonas cercanas, se aporta esta nueva cuadrícula por ser una especie muy escasa y rara en la CAPV y por estar separada de las manchas hasta ahora conocidas y muy cerca del límite provincial con Araba, donde no es descartable su presencia. En la nueva localidad fueron contabilizados más de 50 individuos.

Cita importante para la CAPV donde la especie está incluida bajo el epígrafe "En peligro crítico de extinción" (CR) de la Lista Roja de Flora Vascular de la CAPV. (Fig. S14). Especie endémica de la Cordillera Cantábrica, Pirineos, norte del Sistema Ibérico y oeste del Sistema Central.

\section{Rhynchospora alba (L.) Vahl.}

BIZKAIA: 30TVN9391, Galdames, 630 m, pequeño trampal, 4.8.2014, A. Prieto.

Especie presente en turberas, esfagnales y brezales higroturbosos sobre suelos silíceos de influencia cantábrica (Aizpuru et al., 1999). Planta escasa en el territorio de Bizkaia donde solamente se conocía de las localidades de Urkiola (Aseginolaza et al., 1984) y de Villaro-Areatza, en el trabajo "Seguimiento estacional de los trampales de Larreder, P.N. Gorbea" (Anónimo, 2002). Su tardía fenología, y su porte discreto salvo en época floral, dificultan su localización, por lo que puede ser más abundante que lo que las escasas citas indican.

Salix cantabrica Rech. fil.

BIZKAIA: 30TVN6878, Karrantza Harana/Valle de Carranza, Salduero, 1070 m, borde del regato del Pozo Negro, 17.06.2011, E. Díaz Meñaka, S. Patino, \& I. Aginako; 
30TVN6978, Ibídem, Monte Grande, 970 m, 17.06.2011, S. Patino, E.J. Díaz \& F.J. Martínez; 30TVN6877, Ibídem, 980 m, cabecera de barranco sobre brezales sometidos a fuerte influencia atlántica, 21.08.2013, E.J. Díaz, S. Patino \& J. Valencia.

Arbusto de terrenos higroturbosos y orillas de arroyos sobre sustrato silíceo. Especie incluida en la Lista Roja de la Flora Vascular de la CAPV bajo el epígrafe "Casi amenazada" (NT), es conocida desde hace años de las montañas cercanas de Cantabria y Burgos. La localización de una de estas poblaciones posibilitó su inclusión previa en la Lista Roja de la Flora Vascular de la CAPV (Aizpuru et al., 2010). En las tres localidades citadas, comparte hábitat con S. aurita, especie algo más abundante. De importancia corológica para Bizkaia. Novedad provincial.

Salpichroa origanifolia (Lam.) Baill.

BIZKAIA: 30TVN0096, Leioa, Lamiako, 3 m, 20.09.2014, J. Elorza \& J. Valencia.

Planta originaria de Sudamérica que se encuentra naturalizada en herbazales, setos y riberas de los valles atlánticos. De una cuadrícula cercana fue citada en Patino \& Valencia (2000).

Selaginella kraussiana (G. Kuntze) A. Brau

BIZKAIA: 30TWN2772, Mañaria, Parque Natural de Urkiola, 445 m, borde de pista 26.7.2013, A. Prieto.

En la Comunidad Autónoma del País Vasco se considera especie alóctona naturalizada no invasora principalmente de hábitats naturales y seminaturales (Campos \& Herrera, 2009). En la Comunidad Autónoma del País Vasco se ha citado en 9 cuadrículas UTM de 1 km de lado, tres de ellas en Bizkaia: Mendexa, Ondarroa (Alejandre, 1989) y Gatika (Patino et al., 2009-2010).

Senecio carpetanus Boiss.\& Reuter

ARABA: 30TWN4026, Bernedo, Parque Natural de Izki, 740 m, pasto húmedo 26.8.2011, 6 ejemplares. A. Prieto

Endemismo ibérico de prados inundables de suelos calizos de piso montano. En Izki sólo se tenía constancia de una cita en la cuadrícula WN4424 (Uribe-Echebarría, 2001) en el entorno conocido como el Juncal, cerca de San Román de Campezo. Esta población no ha sido localizada, encontrándose además el hábitat muy modificado, con colonización por parte de arbustos, al parecer porque esa zona ya no es usada por el ganado. Considerada "En peligro de extinción" (EN) en la Lista Roja de la Flora Vascular de la CAPV. 


\section{Sideritis ovata Cav.}

BIZKAIA: 30TVN6487, Karrantza Harana/Valle de Carranza, Pico El Moro, 727-817 m y 30TVN6587, Ibídem, 743-817 m, repisas herbosas del roquedo, sustrato calizo, 05.07.2012, S. Patino.

Endemismo ibérico que aparece en repisas herbosas de roquedos calizos, grietas de lapiaz y claros de quejigal. En Bizkaia solamente se conocía de los municipios de Urduña-Orduña y Galdames. Aparece ahora en el occidente encartado junto al Valle de Soba, de donde fue repartida del monte Mazo Grande en la Exsiccata Selecta de la AHIM (Centuria I, 1996) por los autores del presente artículo. Valorada como "Con menor riesgo de extinción" en la Lista Roja de la Flora Vascular de la CAPV.

Soliva sessilis Ruiz \& Pav.

BIZKAIA: 30TVN9991, Barakaldo, Kareaga, 15 m, grietas de pavimentación en zona urbanizada. 16.05.2014, S. Patino.

Especie introducida de origen Sudamérica y naturalizada en arenales costeros antropizados. Citada de Leioa por Campos \& Herrera (1998), posteriormente se ha vuelto a señalar en el barrio de Ondiz (Fidalgo et al., 2012) en el mismo municipio.

\section{Sorbus domestica L.}

BIZKAIA: 30TWP2504, Gautegiz-Arteaga, Kanala, $9 \mathrm{~m}$, talud de carretera, 09.10.2016, J. Elorza.

Especie que se cultivaba por sus frutos, aparece en setos y lindes, o formando parte de quejigales y carrascales. No se había observado naturalizado en Bizkaia.

\section{Sorbus hybrida L.}

BIZKAIA: 30TVN6877, Karrantza Harana/Valle de Carranza, Salduero. Zalama, $1010 \mathrm{~m}$ brezal argomal junto a hayedo, 17.06.2011, E.J. Díaz, J. Elorza, S. Patino \& J. Valencia.

Se encuentra en claros y orlas de hayedos y robledales de roble albar del piso montano de la vertiente atlántica. De Álava había sido citado como Sorbus x semipinata de la Sierra de Urkilla. De Gipuzkoa se la conoce de la Parzonería de Sierra de Altzania (Apota), donde habita en claros de plantaciones forestales citado por Aizpuru et al., 1996 (cf. Lizaur, 2003). Primera cita vizcaína para esta especie incluida en la categoría "En peligro crítico de extinción" (CR) de la Lista Roja de Flora Vascular de la CAPV. 
Spiranthes aestivalis (Poir.) Rich.

BIZKAIA: 30TWN2368, Zeanuri, Alto de Barazar, 632 m, 4.8.2011, A. Prieto; 30TWN2469, Ibídem, $643 \mathrm{~m}$, terrenos higroturbosos, muy escasos ejemplares en ambas poblaciones, 20.7.2012, A. Prieto; 30TVN7293, Trucios-Turtzioz, 615 m, trampal, 7.7.2015, A. Prieto.

ARABA: 30TWN2361, Zigoitia, Parque Natural Gorbeia, monte Oketa, 770-820 m, 30TWN2260, Ibídem, 715 m; y 30TWN2360, Ibídem, 700 m, terrenos higroturbosos, 18.8.2009, A. Prieto.

La localización de estas importantes poblaciones de Gorbea, ha sido posible gracias a las indicaciones de Jon Maguregi.

Orquídea de turberas y prados húmedos. Se aportan varias localidades para esta especie poco conocida en Bizkaia, donde contaba con las citas de Karrantza Harana/Valle de Carranza, Ranero y Alonsotegi (Aseginolaza et al., 1984), donde también se recoge la del Puerto de Urkiola (Navarro, 1982). Especie incorporada como "Vulnerable" (VU) en la Lista Roja de la Flora Vascular de la CAPV. (Fig. S15).

Trachycarpus fortunei (Hook.) Wendl.

BIZKAIA: 30TWP071024, Gatika, Butroe, 5 m, naturalizada en los alrededores del río Butroe, 12.12.2015, J. Elorza \& S. Patino.

Especie originaria de China utilizada en jardinería en zonas templadas y que a veces se asilvestra. Hasta el momento no contaba con antecedentes en nuestra zona. En esta localidad se pueden observar numerosos ejemplares de diversos tamaños.

\section{Urtica membranacea Poir.}

BIZKAIA: 30TVN7393, Karrantza Harana/Valle de Carranza, Arroyo de los Ladrones, $355 \mathrm{~m}$. entre grandes bloques en zona frecuentada por el ganado, 15.02.2011, E.J. Díaz, S. Patino \& F.J. Martínez.

GIPUZKOA: 30TWN4561, Oñati, Aitzulo, 590 m, terrenos abrigados y nitrogenados dentro del covachón, 29.01.2017, E.J. Díaz, Iker Novoa, S. Patino, J. Valencia, Herbario Digital Xavier de Arizaga, Oñatiko Naturzaliak, Herbario Sestao \& al.

Para Gipuzkoa, las únicas localidades citadas son las de San Sebastián e Irun (Willkomm \& Lange, 1861). Especie de zonas antrópicas, de ambientes nitrogenados y alterados, costeros. Nuevas localidades para esta especie nitrófila escasa, que aparece tanto en localidades costeras como en covachones y oquedades frecuentados por el ganado en algunas de nuestras montañas. 
Utricularia australis $\mathrm{R}$. Br.

ARABA: 30TWN5543, San Millán/Donemiliaga, Bikuña/Vicuña, laguna, 860 m, 23.7.2014, A. Prieto.

Las primeras citas para esta especie en la CAPV se deben a Bubani (1901) que la citó de Algorta, Gredilla (1913) la cita de Vitoria y del río Zadorra y Guinea (1949) del río Gobelas. Posteriormente, Aseginolaza et al. (1984) la citan de Altube y Arreo. Todas ellas refiriéndose a $U$. minor o $U$. vulgaris. De estas poblaciones, sólo se mantiene en Altube, ya que la población de Arreo, aunque ha sido observada en épocas más recientes (Aizpuru et al., 1997), podría haber desaparecido (Monteoliva et al., 2011). Recientemente ha sido citada en otros enclaves: Quintana, Izki (Prieto, 2009) y Uzkiano, Amurrio (Iglesias-Carrasco, 2013). Se añade otra población más en territorio alavés, siguiendo las indicaciones de varios estudios sobre el estado ecológico de algunos humedales (Gobierno Vasco, 2004 y Monteoliva et al., 2013). Especie incluida en la Lista Roja de la Flora Vascular de la CAPV como "En peligro de extinción" (EN).

Vandenboschia speciosa (Willd.) G. Kunkel

BIZKAIA: 30TVN7481, Karrantza Harana/Nalle de Carranza, arroyo Bernales, 370 m, 10.4.2013, A. Prieto; 30TVN7682, Ibídem, Arroyo Las Escaleras, 490 m, 19.3.2013, A. Prieto.

En la Comunidad Autónoma del País Vasco se encuentran dispersas varias poblaciones por Bizkaia y Gipuzkoa (Patino et al., 2001a).

Helecho presente en oquedades rocosas de cascadas de arroyos. En Bizkaia se ha citado de una decena de cuadrículas de 1x1 km, aunque la población de Zierbena se da por extinguida. También ha sido citada de Ondarroa sin especificar UTM. En Ordunte las primeras observaciones de esta especie datan de 1989 donde Alejandre \& Barredo (1990) citan esta especie del arroyo Bernales. En 2006 se cartografía tres pequeños núcleos de Trichomanes en ese mismo arroyo (Prieto, 2006; Otxoa et al., 2007). En este trabajo se recogen otras dos poblaciones. Una de ellas se ubica también en el mismo arroyo, aguas arriba de las tres ya conocidas y la otra se ha localizado en el arroyo Las Escaleras. Considerada en la Lista Roja de la Flora Vascular de la CAPV como "Vulnerable" (VU).

Verbena litoralis var. brevibracteata (Ktze) N. O'Learly

BIZKAIA: 30TWP0702, Gatika, zona cercanuna al río Butroe, $6 \mathrm{~m}$, herbazales ruderalizados junto al castillo de Butroe, 13.01.2014, J. Elorza \& S. Patino; 30TWN0094, Sestao, río Galindo, 4 m, terrenos ruderales sobre el río Galindo, 07.06.2011, S. Patino; 30TVN8991, Galdames, El Cuadro, 125 m, terrenos alterados junto a antigua vía de Galdames, S. Patino \& J. Valencia. 
Alóctona originaria de Sudamérica, presente en márgenes de ríos y arroyos sobre terrenos ruderalizados. Se aportan tres nuevas citas para esta alóctona americana descubierta recientemente en nuestra zona y que ayudarán a perfilar su área de distribución. Hasta el momento, solamente contaba con las citas publicadas en Patino et al. (2009-2010), donde se señala de los municipios de La Cuadra y Valle de Trápaga-Trapagaran.

Veronica sennenii (Pau) M.M. Mart. Ort. \& E. Rico

BIZKAIA:30TVN6586, Karrantza Harana/Valle de Carranza, Peña El Moro, 550 m, pasto pedregoso calizo, 12.05.2015, S. González Ahedo.

Especie indiferente al sustrato, aunque preferentemente calcícola, vive en pastos pedregosos, claros forestales y crestones venteados, aunque aparece a veces en arenales costeros. Planta rara en la vertiente cantábrica, sólo conocida de Bizkaia en Urduña-Orduña (Aseginolaza et al., 1984, donde fue citada como Veronica orsiniana Ten.), y Galdames, Pico La Cruz (Patino et al., 2009-2010).

\section{Woodwardia radicans $(\mathrm{L}.) \mathrm{Sm}$.}

BIZKAIA: 30TVN8490, Sopuerta, Olabarrieta, 208 m, sobre taludes húmedos y sombríos, en barranco encajado, con grandes bloques de arenisca y numerosas cascadas, 10.01.2013, E.J. Díaz \& S. Patino; 30TVN8390, Ibídem, La Cilla, 117 m, ladera rezumante y cubierta de vegetación en zona orientada al norte, 23.05.2012, E.J. Díaz \& S. Patino; 30TVN8391, Ibídem, Alen, 406 m, barranco encajado de la cara norte de Alen, 15.02.2011, E.J. Díaz \& S. Patino; 30TVN9988, 30TWN0087, 30TVN8391, Alonsotegi, La Llana, 75-80 m y 35-75 m, en barranco encajado muy resguardado, sustrato silíceo, 04.03.2011, E.J. Díaz, I. Aguinaco \& S. Patino; 30TVN8893, Galdames, Pozo Vinagre, 150 m, S. Patino \& E.J. Díaz; 30TVN9786 y 30TVN9787, Güeñes, La Quadra, arroyo Markoleta, 25-465 m, taludes de arroyo encajado, húmedo y sombrío, 22.03.2008, S. Patino, E. Miguel \& T. Oyanarte; 30TWN4590, Markina-Xemein, Larruskain, 208-245 m, en orillas del arroyo, corren peligro debido a la degradación de la zona, 02.10.07, S. Patino \& J. Valencia; 30TWP1504 y 30TWP1505, Bakio y Mungia, arroyos Estepona y Emerando, 2-83 m, muy abundante en taludes y márgenes del río con aliseda bien conservada, 30.12.2013, S. Patino; 30TVN7178, Karrantza Harana/Valle de Carranza, arroyo Peñaranda, 460 m, escasos ejemplares en la margen del río, 2.4.2013, A. Prieto; 30TVN7489, Karrantza Harana/Valle de Carranza, río Zarangustillo (La Jilguera), 415 m, escasos ejemplares en la margen del río, F.J. Martínez.

Helecho presente en barrancos poco expuestos, sombríos y húmedos, en cascadas y arroyos. En la Comunidad Autónoma del País Vasco se ha localizado en los tres 
territorios (Patino et al., 2001a), siendo más abundante en Bizkaia, donde se ha citado de 57 cuadrículas de 1x1 km, concentrándose principalmente en la parte central del litoral y en las Encartaciones. Estas nuevas localidades que presentamos ayudan a perfilar su área en nuestro territorio. Es una especie catalogada como "Vulnerable" (VU) en la Lista Roja de la Flora Vascular de la CAPV.

\section{Material Suplementario:}

http://www.aranzadi.eus/fileadmin/docs/Munibe/flora_vascular_xiii.pdf

\section{Bibliografía}

Aedo, C., Herrá, C., Gallo, M.L., Escallada, E.L., Moral, G.M., Patallo, J. 1986. Contribuciones al conocimiento de la flora montañesa, V. An. Jardín Botánico Madrid 43(1): 57-64.

Aedo, C., Aldasoro, J.J., Argüelles, J.M., Díaz Alonso, J.L., González del Valle, J.M., Herrá, C., Laínz, M., Moreno Moral, G., Patallo, J., Sánchez Pedraja, Ó. 1993. Contribuciones al conocimiento de la flora cantábrica. Fontqueria 36: 349-374.

Aedo, C., Aldasoro, J.J., Arguelles, J.M., Carlón, L., Díez Riol, A., González del Valle, J.M., Laínz, M., Moreno Moral, G., Patallo, J., Sánchez Pedraja, Ó. 2000. Contribuciones al conocimiento de la flora Cantábrica: 4. Bot. Cienc. Nat. IDEA 46: 7-119.

Agut, A. 2012. Estudio sobre el impacto ecológico de Azolla filiculoides y la eficacia de los métodos físicos de control y erradicación como medida de gestión de las poblaciones de esta especie en los ecosistemas acuáticos del municipio de Vitoria-Gasteiz (Fase I). Departamento de Medio Ambiente y Espacio Público del Ayuntamiento de Vitoria-Gasteiz.

Aizpuru, I., Aparicio, J.M., Aperribay, J.A., Aseginolaza, C., Elorza, J., Garín, F., Patino, S.,Pérez Dacosta, J.M., Pérez de Ana, J.M., Uribe-Echebarría, P.M., Urrutia, P., Valencia, J., Vivant, J. 1996. Contribuciones al conocimiento de la flora del País Vasco. An. Jardín Botánico Madrid 54(1): 419-435.

Aizpuru, I., Aperribay, J.A., Aseginolaza, C., Garín, F., Vivant, J. 1997. Contribuciones al conocimiento de la flora del País Vasco. Munibe, Cienc. Nat. 49: 65-76.

Aizpuru, I., Aseginolaza, C., Garín, F., Vivant, J. 1998. Contribuciones al conocimiento de la flora del País Vasco III. Munibe, Cienc. Nat. 50: 7-19.

Aizpuru, I., Aseginolaza, C., Uribe-Echebarría, P.M., Urrutia, P., Zorrakin, I. 1999. Claves ilustradas de la flora del País Vasco y territorios limítrofes. Gobierno Vasco. Vitoria-Gasteiz.

Aizpuru, I., Tamaio, I., Uribe-Echebarria, P.M., Garmendia, J., Oreja, L., Balentzia, J., Patino, S., Prieto, A., Campos, J.A., Biurrun, I., Garcia-Mijangos, I., Herrera, M. 2010. Lista Roja de la flora vascular de la CAPV. IHOBE, Departamento de Medio Ambiente Planificación territorial Agricultura y Pesca del Gobierno Vasco. Vitoria-Gasteiz. 
Alejandre, J.A., Aseginolaza, C., Gómez, D., Montserrat, G., Morante, G., Uribe-Echebarría, P.M., Urrutia, P., Zorrakin, I. 1987. Adiciones y correcciones al catálogo florístico de Álava, Vizcaya y Guipúzcoa. Munibe, Cienc. Nat. 39: 123-131.

Alejandre, J.A. 1989. Datos corológicos sobre pteridófitos peninsulares. Fontqueria 24: 3-4.

Alejandre, J.A., Barredo, J.J. 1990. Mapa 68 (adiciones). En: Asientos para un atlas corológico de la flora occidental, 16. J. Fernández Casas (Ed.). Fontqueria 28: 65-186.

Alejandre, J.A., López, J.M.G., Sanz, G.M. 2009. Atlas de flora vascular de Burgos. Junta de Castilla-León, Caja Rural de Burgos.

Allorge, V., Allorge, P. 1941. Plantes rares ou intéressantes du NW de l'Espagne, principalement du Pays Basque. Bull. Soc. Botanique de France 88: 226-254.

Anónimo. 2002. Seguimiento estacional de los trampales de Larreder, Parque Natural del Gorbea (Bizkaia). Gobierno Vasco. Vitoria-Gasteiz.

Aseginolaza, C., Gómez, D., Lizaur, X., Montserrat-Martí, G., Morante, G., Salaverria, M. R., Uribe-Echebarría, P. \& Alejandre, J.A. 1984. Catálogo florístico de Álava, Vizcaya y Guipúzcoa. Gobierno Vasco, Viceconsejería de Medio Ambiente. Vitoria-Gasteiz.

Bariego, J.P., Gastón, A. 2002. Catálogo florístico de los Montes de Ordunte (Burgos, España). Ecología 16: 97-152.

Barredo Pérez, J.J. 2005. Hallazgo de plantas nuevas o raras en Vizcaya. Munibe, Cienc. Nat. 56: 49-54.

Bubani, P. 1901. Flora Pyrenaea per ordines naturales gradatim digesta. Ed. Ulricus Hoeplius. Mediolani.

Cadiñanos, J.A., Fidalgo, E., Llorente, A. 2010. Aportaciones a la flora vascular de Vizcaya, Guipúzcoa y Cantabria (III). Munibe, Cienc. Nat. 58: 31-38.

Campos, J.A., Berastegi, A., Darquistade, A. 2002. Sobre algunas plantas poco conocidas del País Vasco y zonas limítrofes. Estudios Museo Cienc. Nat. Álava 17: 125-130.

Campos, J.A., Herrera, M. 1998. Datos sobre la flora vascular introducida en el País Vasco y Cantabria oriental. Lazaroa 19: 71-84.

Campos, J.A., Herrera, M. 2009. Diagnosis de la flora alóctona invasora. Departamento de Medio Ambiente y Ordenación del Territorio, Gobierno Vasco. Vitoria-Gasteiz.

Carlón, L., González del Valle, J.M., Laínz, M., Moreno Moral, G., Rodríguez Berdasco, J.M., Sánchez Pedraja, O. 2010. Contribuciones al conocimiento de la flora cantábrica VIII. Jardín Botánico Atlántico 7: 1-96.

Castroviejo, S. (coord.) 1986-2015. Flora ibérica: Plantas vasculares de la Península Ibérica y Baleares. Real Jardín Botánico (CSIC). Madrid.

Duran, J.A. 2014. Catálogo de la flora vascular de Cantabria. Monografías de Botánica Ibérica 13. Editorial Jolube Consultoría Ambiental. Jaca. 
EKOS 2005. Cartografía de flora amenazada en la Reserva de la Biosfera de Urdaibai. Patronato de la Reserva de la Biosfera de Urdaibai - Gobierno Vasco. Informe inédito.

Fidalgo, E., Llorente, A., Cadiñanos, J.A., Tarruella, J. 2012. Aportaciones a la flora vascular del norte de la Península Ibérica. Munibe, Cienc. Nat. 60: 7-38.

Gandoger, M. 1917. Catalogue des plantes récoltées en Espagne et en Portugal pendant mes voyages de 1894 à 1912. Hermann, L'Home, Masson libraires. Paris.

Gredilla, A.F. 1913. Apuntes para la corografía botánica vasco-navarra. En: Geografia general del País Vasco-Navarro. Carreras \& Candi (Ed.): 459-567. A. Martín. Barcelona.

Guinea, E. 1949. Vizcaya y su paisaje vegetal (Geobotánica vizcaína). Junta de Cultura de Vizcaya. Bilbao.

Guinea, E. 1953. Geografía botánica de Santander. Diputación provincial de Santander. Santander.

Gobierno Vasco. 2004. Red de seguimiento de la calidad ecológica de los humedales interiores de la Comunidad Autónoma del País Vasco. Fundación General de la Universidad Autónoma de Madrid.

Iglesias-Carrasco, M. 2013. Nueva cita de Utricularia australis R. Br. en el País Vasco. Munibe, Cienc. Nat. 61: 149-152.

Laínz, M. 1963. Aportaciones al conocimiento de la flora cántabro-astur VII. Bol. Inst. Estud. Asturianos, Supl. Ci. 7: 35-81.

Lizaur, X. 2003. Actualización (suplemento) del “Araba, Bizkaia eta Gipuzkoako landare katalogoa- Catálogo florístico de Álava, Bizkaia y Gipuzkoa. Gobierno Vasco. Araba y Gipuzkoa.

Lizaur, X., Salaverría, M.R. 1987. Adiciones al Catálogo florístico de Álava, Vizcaya y Guipúzcoa (Aseginolaza et al., 1984). Munibe, Cienc. Nat. 39: 115-118.

Lorda López, M. 2001. Flora del Pirineo Navarro. Guineana 7: 1-527.

Lorda López, M. 2013. Catálogo Florístico de Navarra. Editorial Jolube Consultoría Ambiental. Jaca.

Llorente, A., Cadiñanos, J.A., Fidalgo, E. 2009. Aportaciones a la flora vascular de Vizcaya, Guipúzcoa y Cantabria. Munibe, Cienc. Nat. 57: 47-65.

Monteoliva, A., Monteoliva, J.A., Alonso, G., del Pozo, R., Barquilla, M. 2011. Red de seguimiento del estado ecológico de los humedales interiores de la Comunidad Autónoma del País Vasco. Estado ecológico ciclo 2009/2010. Informe inédito realizado por Ecohydros S. L. para Agencia Vasca del Agua.

Monteoliva, A., Monteoliva, J.A., Alonso, G., del Pozo, R., Barquilla, M. 2013. Red de seguimiento del estado ecológico de los humedales interiores de la Comunidad Autónoma del País Vasco. Estado ecológico ciclo 2011/2012. Informe inédito realizado por Ecohydros S. L. para Agencia Vasca del Agua. 
Navarro, M.C. 1982. Contribuciones al estudio de la Flora y Vegetación del Duranguesado y la Busturia (Vizcaya). Tesis doctoral. Universidad Complutense de Madrid. Madrid.

Onaindia, M. 1986. Ecología vegetal de las Encartaciones y Macizo del Gorbea (Vizcaya). Universidad del País Vasco - E.H.U. Bilbao.

Otxoa, E., Oyanarte, T., Patino, S., Valencia, J., Miguel, E., Prieto, A., Elorza, J. 2007. Cartografiado a escala 1:5000 de las poblaciones vizcaínas de plantas incluidas en el catálogo vasco de especies amenazadas, Fase III. Informe inédito realizado para Gobierno Vasco. Vitoria-Gasteiz.

Patino, S., Uribe-Echebarría, P.M., Urrutia, P. \& Valencia, J. 1990. Notas corológicas sobre la flora vascular del País Vasco y aledaños (IV). Estudios Museo Cienc. Nat. Álava 5: 77-81.

Patino, S., Uribe-Echebarria, P. M., Valencia, J., Urrutia, P. 1991. Notas corológicas sobre la flora vascular del País Vasco y aledaños, V. Estudios Museo Cienc. Nat. Álava 6: 57-68.

Patino, S., Valencia, J. 1989. Nuevas aportaciones al catálogo florístico de la Comunidad Autónoma Vasca. Estudios Museo Cienc. Nat. Álava 4: 77-84.

Patino, S., Valencia, J. 2000. Notas corológicas sobre la flora vascular del País Vasco y aledaños (IX). Estudios Museo Cienc. Nat. Álava 15: 221-238.

Patino, S., Valencia, J., Prieto, A., Elorza, J. 2001a. Helechos paleotropicales de la Comunidad Autónoma Vasca: Situación actual y algunos apuntes para su conservación. Sociedad de Ciencias Naturales de Sestao. Informe inédito realizado para Gobierno Vasco. Vitoria-Gasteiz.

Patino, S., Valencia, J., Prieto, A., Elorza, J. 2001b. Estudio Botánico de itinerarios didácticos de los montes Lucero, Serantes y Montaño. Sociedad de Ciencias Naturales de Sestao. Informe inédito realizado para Gobierno Vasco. Vitoria-Gasteiz.

Patino, S., Valencia, J., Miguel, E., Prieto, A., Elorza, J., Oyanarte, T., Toral, R., Otxoa, E., Díaz, E. 2009-2010. Notas Corológicas sobre la flora vascular del País Vasco y aledaños (XII). Estudios Museo Cienc. Nat. Álava 23: 91-111.

Patino, S., Valencia, J., Elorza, J., Miguel, E., Otxoa, E., Díaz, E., Orrantia, O. \& Prieto, A. 2013. Actualización de la cartografía a escala 1:5000 de las poblaciones vizcaínas de plantas incluidas en el Catálogo Vasco de Especies Amenazadas. Informe inédito realizado para el Gobierno Vasco. Sociedad de Ciencias Naturales de Sestao. Sestao.

Pérez de Ana, J.M. 2003. Nuevas citas de flora amenazada y escasa en las Encartaciones (oeste del País Vasco). Estudios Museo Cienc. Nat. Álava 18-19: 69-80.

Prieto, A. 2000. Orquídeas de Bizkaia. Colección Temas Vizcaínos-BBK. Bilbao.

Prieto, A. 2006. Plan de gestión de flora y hábitats (excepto hábitats higroturbosos). En: Plan de gestión del LIC de Ordunte. BASOINSA S.L. Informe inédito realizado para la Diputación Foral de Bizkaia. Bilbao.

Prieto, A. 2009. Estudio de la situación de la flora amenazada en los espacios naturales protegidos de Álava y propuesta de pautas de gestión. Informe inédito realizado para la Diputación Foral de Álava. Vitoria-Gasteiz. 
Ruiz Campo, R. 2007. Flora de nuestro entorno. Sociedad micológica de Portugalete. Zizak 4: 89-91.

Ruiz Campo, R. 2013. Flora de nuestro entorno VI. Sociedad micológica de Portugalete. Zizak 9: 93-97.

Sánchez Martínez, C., Valdeolivas, G. 1995. Guía de la fauna y flora de un municipio cantábrico: Camargo. El Abra Ed., Camargo.

Sennen, F. 1929. Quelques espèces adventices, subspontanées ou cultivées en Espagne et dans le domaine méditerranéen. Cavanillesia 2: 10-42.

Silván, F., Campos, J.A. 2002. Estudio de la Flora Vascular amenazada de los arenales del País Vasco. Departamento de Agricultura y Pesca del Gobierno Vasco. Vitoria-Gasteiz.

Uribe-Echebarría, P.M. 2001. Notas corológicas sobre la flora vascular del País Vasco y aledaños, X. Estudios Museo Cienc. Nat. Álava 16: 93-101.

Van Der Sluys, M., Gónzalez Artabe, J. 1982. Orquídeas de Navarra. Diputación Foral de Navarra.

Willkomm, M., Lange, J. 1870. Prodronnis Florae Hispanicae. Ed. Schweizerbart. Stuttgart. (Reimpresión 1972).

Willkomm, H.M., Lange, J.M.Ch. 1865. Prodromus Florae Hispanicae 2: 1865-1870.

Fecha de recepcion/Date of reception: 23/06/2017 Fecha de aceptacion/Date of acceptance: 30/01/2018 Editor Asociado/Associate editor: Ricardo Ibáñez 